\title{
Intravascular lymphoma as a Potential Cause of Recurrent Embolic Stroke of Undetermined Source
}

\author{
Kyung Ah Woo \\ Dallah Yoo \\ Keun-Hwa Jung \\ Department of Neurology, \\ Seoul National University Hospital, \\ Seoul, Korea
}

Received March 11, 2019

Revised April 2, 2019

Accepted April 2, 2019

\section{Correspondence}

Keun-Hwa Jung, MD, PhD

Department of Neurology,

Seoul National University Hospital,

101 Daehak-ro, Jongno-gu,

Seoul 03080, Korea

Tel +82-2-2072-4901

Fax +82-2-3672-7553

E-mail jungkh@gmail.com
Dear Editor,

Embolic stroke of undetermined source (ESUS) refers to nonlacunar cryptogenic infarcts for which embolism is the suspected mechanism. ${ }^{1}$ Patients with intravascular lymphoma (IVL) frequently develop cerebral occlusive ischemia caused by the intravascular foci of malignant lymphocytes. While other forms of B-cell lymphoma are known to cause stroke-like encephalopathy, ischemia in IVL is unique in that it may closely mimic typical embolic strokes. ${ }^{2,3}$ However, the diagnosis is often missed since previous investigations of ESUS have focused on cardiovascular sources and cancer-related hypercoagulability. ${ }^{4,5}$ We report two cases of IVL that developed recurrent ESUS as the initial and main manifestations.

Patient 1 presented with multistage bilateral cerebral infarctions at the age of 60 years. Three weeks after discharge while on aspirin and clopidogrel, dysarthria aggravated and new multifocal infarctions occurred (Fig. 1A, B). She subsequently developed a high fever without an infection focus, and her serum lactate dehydrogenase (LDH) was elevated to 517 IU/L.

Patient 2 was aged 52 years and developed acute right-hand weakness and visual disturbance over a 5-month period. Conventional brain magnetic resonance imaging (MRI) revealed focal hyperintense T2-weighted lesions in the left splenium and right occipital lobe. Over the next 2 months, acute occlusive infarction of the right middle cerebral artery (MCA) (Fig. 1C), right posterior cerebral artery, and left MCA territories occurred in a sequential manner (Fig. 1D, E) despite receiving combined treatment with aspirin and warfarin. Abdominal computed tomography (CT) showed wedge-shaped infarctions in the spleen and kidneys (Fig. 1F). Sonography revealed deep-vein thrombosis in the bilateral tibial veins. Her serum LDH level was 393 IU/L.

Conventional MRI was performed upon each presentation of stroke using both diffusionweighted imaging and angiography. No intra- or extracranial stenosis was found. Routine laboratory tests, a rheumatological panel, and an extensive cardiac investigation revealed no embolism sources. Contrast-enhanced CT of the chest, abdomen, and pelvis showed no evidence of cancer.

The high fever in patient 1 provoked suspicion of B symptoms, while the recurrent embolic strokes with extraneural involvement in patient 2 raised suspicion of IVL. Cerebrospinal fluid (CSF) polymerase chain reaction analysis was performed to search for a monoclonal lymphoid population. T-cell receptor (TCR) $-\gamma$ rearrangement analysis in patient 1 and immunoglobulin heavy chain $(\mathrm{IgH})$ rearrangement analysis in patient 2 demonstrated monoclonality, suggesting that lymphoid malignancy was present in the central nervous system (CNS).

A brain biopsy confirmed IVL in patient 1 (Supplementary Fig. 1 in the online-only Data Supplement), with atypical large lymphocytes sequestered to the intravascular spaces. Immunohistochemical analysis revealed positive expression of CD20, Bcl-2, Bcl-6, Ki-67, and MUM-1. Chemotherapy was started, but multiorgan failure led to death 6 months later.

@ This is an Open Access article distributed under the terms of the Creative Commons Attribution Non-Commercial License (https://creativecommons.org/licenses/by-nc/4.0) which permits unrestricted non-commercial use, distribution, and reproduction in any medium, provided the original work is properly cited. 

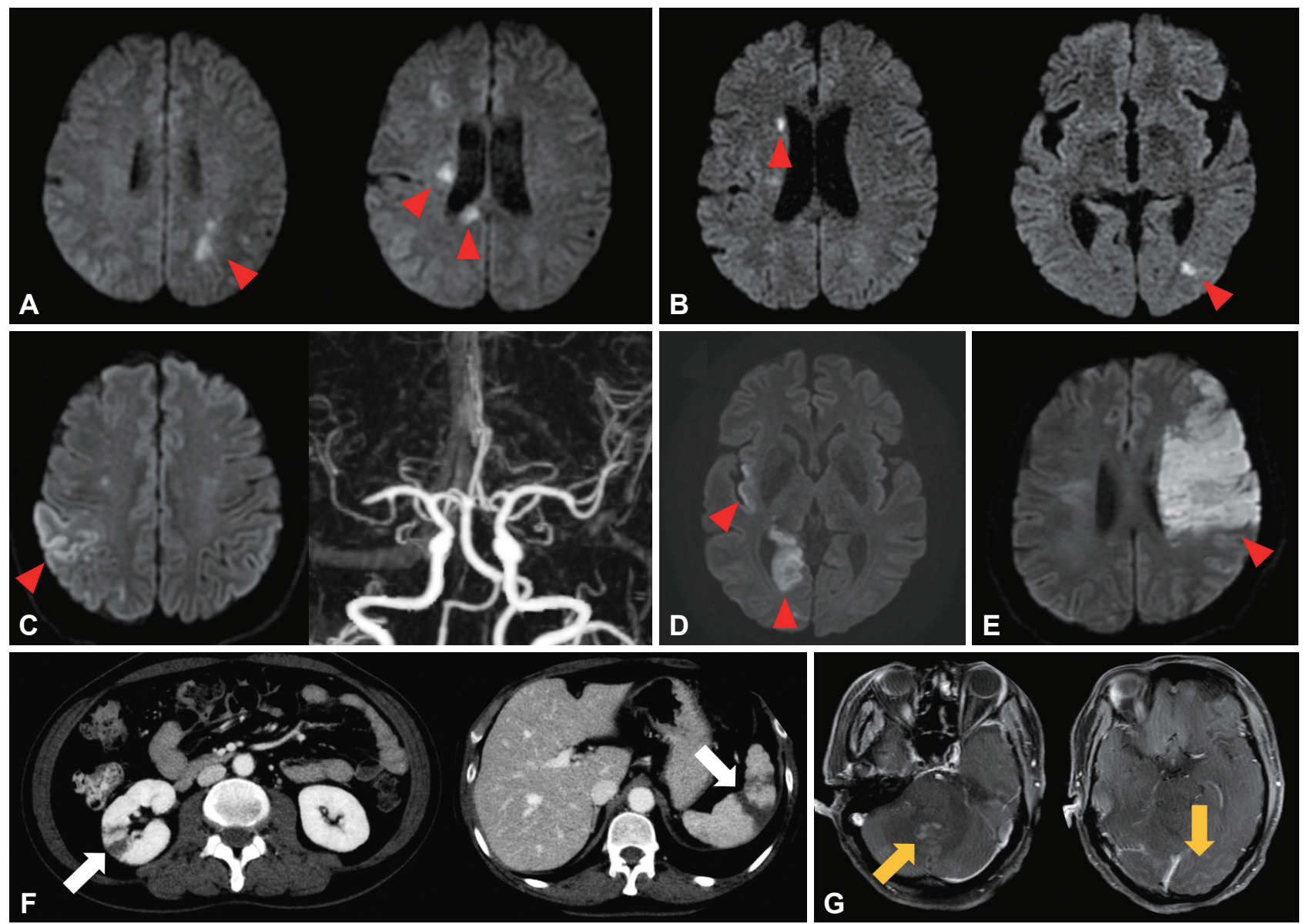

Fig. 1. Brain MRI and systemic radiological features of patients 1 and 2. A-E: Serial brain diffusion-weighted MRI sequences of patient 1 (A, B) and patient 2 (C-E) performed after each episode of acute neurological deterioration. The images revealed multifocal diffusion-restricted lesions with low apparent diffusion coefficient values suggesting acute infarctions (red arrowheads) in the bilateral cerebral hemispheres. In patient 2, CT of the abdomen (F) revealed renal and splenic infarctions (arrows). T1-enhanced MRI of the brain (G) revealed prominent leptomeningeal enhancement in bilateral parieto-occipital regions and nodular enhanced lesions around the fourth ventricle (arrows).

The caregivers of patient 2 refused a biopsy. She underwent cycles of rituximab-combined chemotherapy. MRI performed 5 months later revealed leptomeningeal enhancement with enhanced nodules around the fourth ventricle, which was consistent with meningeal involvement of IVL (Fig. 1G). Her neurological status remained unchanged at the last follow-up.

IVL is a rare form of systemic B-cell lymphoma, with an incidence of less than 1 per million in the general population. ${ }^{6}$ The disease is characterized by selective tropism of malignant lymphocytes within the lumina of small vessels. IVL can involve any organ, but the most commonly affected sites are the skin and CNS, followed by the spleen, lung, and bone marrow. ${ }^{7}$ However, diagnosing IVL is challenging when patients present with neurological complications. The literature indicates that $60 \%$ of CNS IVL cases are diagnosed postmortem. ${ }^{2}$ There is no pathognomonic radiological feature that differentiates CNS IVL from typical embolic strokes, which hinders prompt detection. Although rare in the general population, the lethal course of IVL means that it should not be overlooked when evaluating ESUS.

A tissue biopsy is required to confirm IVL. ${ }^{8}$ This complicates the process of diagnosing CNS IVL, since a brain biopsy sample might not be available, or its results might be inconclusive. We utilized CSF IgH/TCR- $\gamma$ rearrangement analysis to support the diagnosis in our ESUS patients. Molecular analysis is known to be helpful in IVL, although its diagnostic power remains to be further evaluated.'

The present two cases highlight the importance of aggressively investigating IVL in ESUS. Various systemic manifestations of the disease should be carefully searched for, including organ ischemia, hepatosplenomegaly, and B symptoms. CSF monoclonality analysis should be considered for suspected cases, since the presence of monoclonal lymphoid proliferation in CSF may support the diagnosis. 
analysis of 654 patients. BMC Neurol 2016;16:9.

\section{Supplementary Materials}

The online-only Data Supplement is available with this article at https://doi.org/10.3988/jcn.2019.15.3.415.

\section{Conflicts of Interest}

The authors have no potential conflicts of interest to disclose.

\section{Acknowledgements}

This study was approved by Seoul National University Hospital Institutional Review Board for access to the cohort data registry of cerebral infarction and analysis of clinical data, imaging studies, and laboratory results.

\section{REFERENCES}

1. Hart RG, Catanese L, Perera KS, Ntaios G, Connolly SJ. Embolic stroke of undetermined source: a systematic review and clinical update. Stroke 2017;48:867-872.

2. Fonkem E, Dayawansa S, Stroberg E, Lok E, Bricker PC, Kirmani B, et al. Neurological presentations of intravascular lymphoma (IVL): meta-
3. Kim JM, Jung KH, Lee ST, Park HK, Chu K, Roh JK. Stroke mimicking encephalopathy as an initial manifestation of diffuse large B-cell lymphoma. J Clin Neurol 2009;5:97-100.

4. Cestari DM, Weine DM, Panageas KS, Segal AZ, DeAngelis LM. Stroke in patients with cancer: incidence and etiology. Neurology 2004; 62:2025-2030.

5. Bick RL. Cancer-associated thrombosis. N Engl J Med 2003;349:109111.

6. Zuckerman D, Seliem R, Hochberg E. Intravascular lymphoma: the oncologist's "great imitator." Oncologist 2006;11:496-502.

7. Fonkem E, Lok E, Robison D, Gautam S, Wong ET. The natural history of intravascular lymphomatosis. Cancer Med 2014;3:1010-1024.

8. Shimada K, Kinoshita T, Naoe T, Nakamura S. Presentation and management of intravascular large B-cell lymphoma. Lancet Oncol 2009;10: 895-902.

9. Baehring JM, Longtine J, Hochberg FH. A new approach to the diagnosis and treatment of intravascular lymphoma. J Neurooncol 2003; 61:237-248. 